\title{
Influencia de los defecaderos de camélidos sobre el desarrollo vegetal y riqueza de especies en morrenas glaciales, Tierra del Fuego
}

\author{
Camelid defecation influences vegetation development and species richnesss on glacial \\ moraines, Tierra del Fuego
}

JUAN M. HENRÍQUEZ1

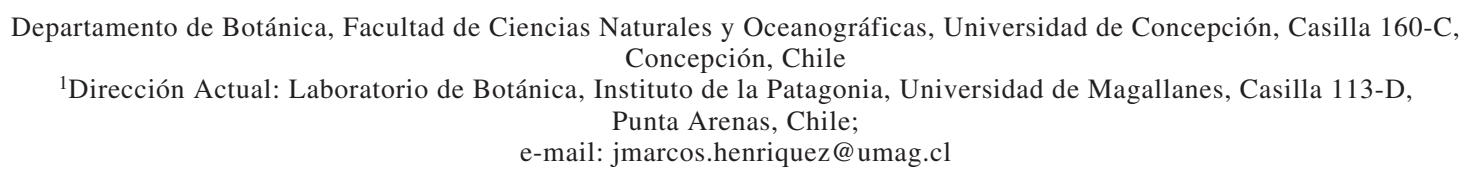

\section{RESUMEN}

Los escasos trabajos de interacción planta-herbívoro en ambientes severos han concluido que los herbívoros tienen un rol menor sobre el proceso de colonización en los estados tempranos de la sucesión. Aquí se documentan los efectos de defecaderos de un herbívoro ungulado (Lamma guanicoe) sobre los patrones vegetales en una intermorrena glacial reciente. Se evaluó la composición florística, frecuencia y síndrome de dispersión de las especies presentes sobre los defecaderos y sitios control. Parámetros de suelo fueron analizados en ambos substratos. La riqueza y diversidad de especies vasculares fueron significativamente mayores sobre los defecaderos. El síndrome de dispersión dominante es la anemocoría en la vegetación de ambos tipos de substratos. Los defecaderos presentan valores superiores de materia orgánica, nitrógeno y fósforo, indicando condiciones localmente más favorables para el establecimiento de plántulas en este ambiente pobre en nutrientes y materia orgánica. Finalmente, se discute acerca de los posibles mecanismos que originan la mayor riqueza y diversidad de especies sobre los defecaderos de guanaco, concluyendo que constituyen una fuente potencial de propágulos que puede acelerar el proceso de colonización en morrenas glaciares.

Palabras clave: defecaderos, síndrome de dispersión, Lamma guanicoe, sucesión primaria.

\begin{abstract}
The few studies of herbivore-plant interaction in severe environments have concluded that the herbivores have a minor role on the process of colonization in the early states of the succession. This paper describes the effects of camelid (Lamma guanicoe) dung heaps on vegetation patterns on a recent glacial moraine. Floristic composition, diversity, abundance and dispersion syndrome spectra of the vegetation growing on dung heaps was compared with that of control sites unaffected by camelid defecation. Soil parameters of both substrates were analyzed. Vascular species riches and diversity were significantly higher on dung heaps. Anemochory was the dominant dispersion syndrome on both substrate types. Dung heaps had higher levels of organic matter, nitrogen and phosphorus, suggesting more favorable substrate conditions. Thus, dung heaps appear to offer favorable sites for seedling establishment in an otherwise resource-poor environment, and their role as seed sources could accelerate the process of colonisation of glacial moraines.
\end{abstract}

Key words: dung heaps, dispersion syndrome, Lamma guanicoe, primary succession. 


\section{INTRODUCCIÓN}

La colonización de terrenos recientemente expuestos producto del retroceso de ventisqueros es controlada por diversos factores y procesos biológicos (Crocker \& Major 1955, Chapin 1993). La presencia de grandes herbívoros y su posible influencia sobre el proceso de establecimiento vegetal en estos ambientes es un factor biótico aún escasamente investigado (Matthews 1992).

Históricamente los herbívoros han sido considerados solo ejerciendo un rol negativo sobre el establecimiento vegetal, por lo tanto importantes agentes de disturbio a través del pastoreo, pisoteo y excavaciones (Pickett \& White 1985, Meadows \& Meadows 1991). Considerando su impacto negativo, Walker \& Chapin (1987) señalan que la herbivoría tendría un rol menor en el proceso de colonización en ambientes severos. Los escasos trabajos de interacción planta-herbívoro en ambientes glaciales concluyen que estos tendrían un rol menor en la distribución de propágulos (Dollenz 1991, Chapin 1993), pero mayor en la mortalidad de plántulas en los estados sucesionales primarios (Edwards \& Gillman 1987).

Es concebible que los herbívoros también tengan algunos efectos positivos en el establecimiento vegetal de los terrenos recientemente deglaciados; sin embargo esta posibilidad ha recibido menos atención. El ingreso de semillas y nutrientes en fecas son dos posibles aportes positivos en sistemas en donde ambos son escasos. Los escasos estudios en valles glaciares señalan la anemocoría como el principal síndrome de dispersión de semillas (Matthews 1992). Recientes estudios han documentado un rol más significativo de los herbívoros, especialmente por endozoocoria, en el transporte de semilla en sitios sucesionales (Gill \& Beardall 2001, Pakeman et al. 2002) influyendo sobre la dinámica vegetal (Malo \& Suarez 1995, 1998), aunque no existen estudios similares en ambientes severos. Por otro lado, la presencia de fecas aporta un substrato rico en materia orgánica y nutrientes favoreciendo el desarrollo y evitando la desecación de las plantas (Tilman 1988). Es posible, por lo tanto, esperar que la presencia de fecas en valles glaciares, hipotéticamente, favorecería la incorporación de semillas y establecimiento de plántulas en un medio donde el ambiente es adverso y el suelo escaso, siempre y cuando signifiquen la importación desde sitios sucesionalmente más antiguos.

En Tierra del Fuego, el guanaco (Lamma guanicoe) es el único ungulado nativo (Raedeke 1979) y su presencia en los valles glaciares del lado noreste de la Cordillera de Darwin ha sido documentada (Raedeke 1978). Una interesante característica de su comportamiento, a bajas densidades poblacionales, es la tendencia a defecar habitualmente en el área (Raedeke 1979), formando pequeños depósitos localizados que visitan periódicamente. En pastizales, se ha observado alrededor de los defecaderos una aceleración del desarrollo del suelo y de la sucesión de especies o, en su defecto, el incremento del crecimiento y desarrollo de las plantas presentes en las cercanías (Raedeke 1979, Franklin 1982a). En sitios descubiertos, libres de vegetación y con suelos en desarrollo, se desconoce el impacto que estos defecaderos puedan tener sobre los patrones vegetales.

En el glaciar Nueva Zelandia, Tierra del Fuego, Chile, la presencia de defecaderos de guanacos en las etapas sucesionales tempranas conlleva el desarrollo marcado y diferencial de la vegetación con relación a los sitios control. El presente estudio tiene como objetivo evaluar el posible efecto de los defecaderos de Lamma guanicoe sobre la riqueza y abundancia de plantas vasculares en una intermorrena glaciar recientemente derretida.

\section{MATERIALES Y MÉTODOS}

\section{Área de estudio}

El estudio se realizó en el valle del glaciar Nueva Zelandia $\left(54^{\circ} 42^{\prime} \mathrm{S}, 69^{\circ} 21^{\prime} \mathrm{O}\right)$, Fiordo Parry, en la vertiente norte de la cordillera Darwin en la Isla de Tierra del Fuego, Chile. El clima predominante es de Tundra isotérmica (clasificación de Koeppen), con precipitaciones anuales cercanas a los $1.000 \mathrm{~mm}$, distribuidos uniformemente durante el año sin periodo seco (Pisano 1971). La temperatura media anual a nivel del suelo es de $5,4{ }^{\circ} \mathrm{C}$, siendo el mes más helado Agosto con $-0,8{ }^{\circ} \mathrm{C}$ y el más caluroso diciembre con $12,3{ }^{\circ} \mathrm{C}$ (J.M. Henríquez resultados no publicados).

La geomorfología es de un valle glaciar en $\mathrm{U}$, conformado por morrenas frontales y laterales en proceso de colonización vegetal, producto del derretimiento y la acción erosiva de la masa de hielo. Bosques siempreverdes de Nothofagus betuloides ocupan los sitios sucesionales más antiguos, aunque son frecuentes los bosquetes y brezales mixtos con árboles caducifolios pioneros (principalmente de Nothofagus antarctica) en los sitios intermedios. En los sitios cercanos al frente de hielo arbustos rastreros, herbáceas y suelo descubierto son predominantes. La morrena lateral más reciente, 
donde se realiza la presente investigación, se ubica a menos de $50 \mathrm{~m}$ de la masa de hielo y está conformada principalmente por arena, polvo de arcilla y ripio, la cobertura vegetal no supera el $1 \%$.

\section{Muestreo}

En un plano intermorrénico, entre las masas de hielo cubiertas de ripio y la primera morrena lateral, se registró la existencia de 10 defecaderos de guanaco y la presencia de plantas vasculares presentes creciendo sobre ellos (Fig. 1). Los defecaderos se distribuyen, a distancias no superiores a los $30 \mathrm{~m}$ uno del otro, a lo largo de la intermorrena y son visitados anualmente por los guanacos, aumentando en diámetro de cobertura. En una superficie de 8 hectáreas se registraron un total de tres defecaderos estimando una frecuencia de 0,375 defecaderos por hectárea en el área de estudio.

Con la finalidad de determinar la composición florística y calcular la frecuencia de las especies vasculares presentes, en cada uno de los defecaderos se ubicó al centro un cuadrante de $1 \mathrm{~m}^{2}$, subdividido en 25 cuadrados de 400 $\mathrm{cm}^{2}$. En cada subdivisión se registró las especies presentes calculando un promedio en cada defecadero $(\mathrm{n}=10)$. Se calculó la frecuencia de todas las especies en cada cuadrante. De igual forma se realizó un muestreo aleatorio para registrar la composición florística y frecuencia de especies presentes en los sitios carentes de depósitos de fecas (sitios control). En el área de distribución de los defecaderos $(800$ x $150 \mathrm{~m})$ se sortearon 30 cuadrantes en suelos libres de estos (control). Con los datos de frecuencia, se calculó el índice de diversidad de ShannonWiener para cada uno de los substratos.

La determinación de las especies vasculares se realizó in situ, siendo posteriormente corro- borada en laboratorio. Los síndromes de dispersión de semillas se determinaron de acuerdo a Howe \& Westley (1986) y Villagrán et al. (1986).

\section{Análisis de suelos}

Se tomaron muestras de suelos desde cinco defecaderos y cinco sitios control. Las muestras de suelo en cada substrato corresponden a la mezcla de tres porciones proporcionales tomadas al azar desde distintos puntos del sitio, a una profundidad no superior a $\operatorname{los} 5 \mathrm{~cm}$. Las muestras fueron secadas a temperatura ambiente, para luego ser analizadas en el Laboratorio de Suelos de la Facultad de Agronomía, Universidad de Concepción. Se utilizaron técnicas estandarizadas para cada parámetro de suelo medido: porcentaje de nitrógeno total (extracción con $\mathrm{K}_{2} \mathrm{SO}_{4} 1 \mathrm{~N}$, colorimetría; Longeri 1979), materia orgánica (combustión húmeda, diclomato y colorimetría; Schulte 1995) y retención de agua (Peso seco: ISO 11465, 1993); nitrógeno (extracción en agua-colorimetría; Robarge et al. 1983), fósforo (extracción con bicarbonato de sodio $0.5 \mathrm{M}, \mathrm{pH} 8.5$, colorimetría: NCh 2058, Of 1999) y potasio (extracción con acetato de amonio 1M, pH 7.0-espectrofotometría de emisión, United States Department of Agriculture1996) disponible, y pH (en agua relación 1:2.5: ISO 10390, 1994).

\section{RESULTADOS}

La riqueza de especies vasculares fue mayor en los defecaderos. En el sitio de estudio se identificaron un total de 21 especies de plantas vasculares, ocho de las cuales se presentaron en ambos tipos de substratos (Tabla 1). Los defecaderos presentaron un total de 18 especies,

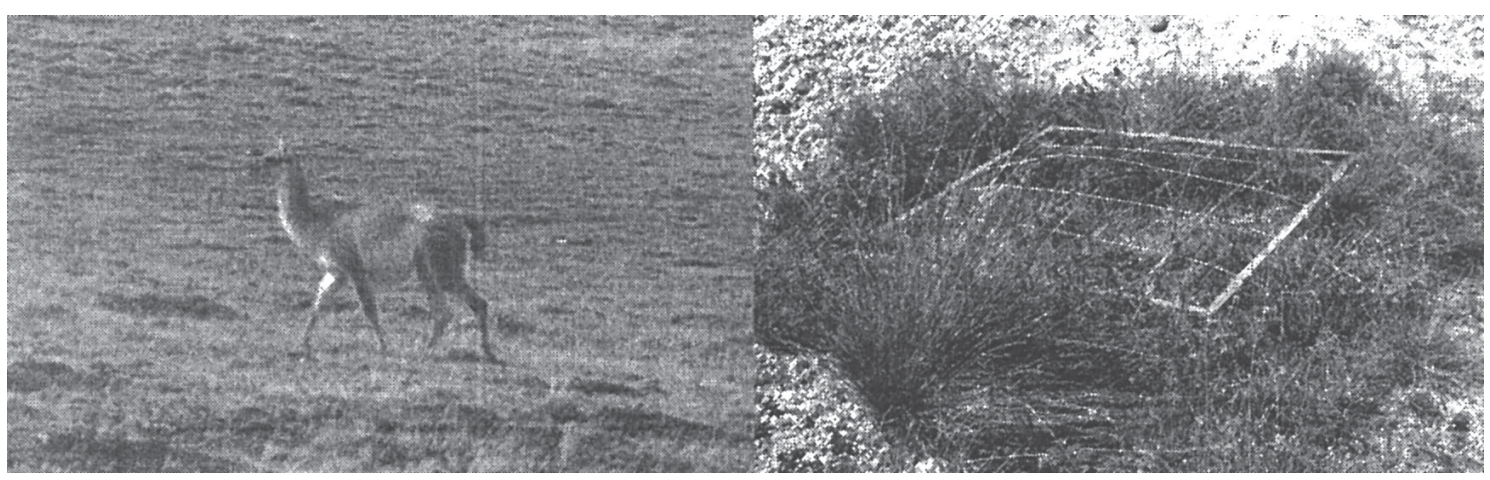

(A)

(B)

Fig. 1: (a) Lamma guanicoe (guanaco). (b) Defecadero de guanaco.

(a) Lamma guanicoe (guanaco); (b) Guanaco dung heaps. 
Listado de especies presentes en los sitios de estudio y su frecuencia promedio en cada sitio (valores por $\mathrm{m}^{2}$ ). Se proporcionan los síndromes de dispersión de cada especie

Checklist of species present at the study sites and their mean frequency (values per $\mathrm{m}^{2}$ ). Dispersion syndromes of each species are shown

\begin{tabular}{|c|c|c|c|}
\hline \multirow[t]{2}{*}{ Especie } & \multicolumn{2}{|c|}{ Sitio } & \multirow{2}{*}{$\begin{array}{c}\text { Síndrome } \\
\text { de dispersión }\end{array}$} \\
\hline & Defecaderos & Arena-ripio & \\
\hline Senecio darwinii Hook. et Arn. & 8,40 & 2,19 & Anemocoria \\
\hline Poa alopecurus Gaudich. Kunth ssp. fueguiana & 14,20 & 0,81 & Anemocoria \\
\hline Gamochaeta spiciformis (Sch.Bip.) Cabr. & 1,40 & 0,00 & Anemocoria \\
\hline Epilobium australe Poepp. et Hausskn. ex Hausskn. & 0,40 & 0,00 & Anemocoria \\
\hline Cardamine glacialis (G.Forster) DC. & 0,40 & 0,00 & Balocoria \\
\hline Cerastium arvense $\mathrm{L}$. & 0,90 & 0,00 & Barocoria \\
\hline Agrostis magellanica Lam. & 0,10 & 0,06 & Anemocoria \\
\hline Acaena magellanica (Lam.) Vahl & 0,70 & 0,00 & Epizoocoria \\
\hline Marsippospermum grandiflorum & 0,90 & 0,16 & Barocoria \\
\hline Gaultheria mucronata (L.f.) Hook. et Arn. & 1,00 & 0,00 & Endozoocoria \\
\hline Luzula alopecurus Desv. & 0,10 & 0,09 & Barocoria \\
\hline Festuca magellanica Lam. & 1,30 & 0,03 & Anemocoria \\
\hline Baccharis nivalis (Wedd.) Sch.Bip. ex Phil. & 1,00 & 1,81 & Anemocoria \\
\hline Geranium magellanicum Hook.f. & 0,10 & 0,00 & Anemocoria \\
\hline Phleum alpinum $\mathrm{L}$. & 3,30 & 0,00 & Anemocoria \\
\hline Empetrum rubrum Vahl ex Will. & 0,20 & 0,00 & Endozoocoria \\
\hline Senecio cuneatus Hook.f. & 0,60 & 0,00 & Anemocoria \\
\hline Gaultheria pumila (L.f.) D.J.Middlenton & 0,40 & 0,31 & Endozoocoria \\
\hline Nothofagus antarctica (G.Forster) Oersted & 0,00 & 0,06 & Anemocoria \\
\hline Gunnera magellanica Lam. & 0,00 & 0,97 & Endozoocoria \\
\hline Luzula chilensis Ness et Meyen ex Kunth & 0,00 & 0,09 & Barocoria \\
\hline Número de especies & 18 & 11 & \\
\hline
\end{tabular}

con un promedio de $6,8 \pm 2,9$ especies $\mathrm{m}^{-2}$ (media \pm DE). Por otro lado, los sitios control presentaron un número total de 11 especies, con un promedio de 1,7 $\pm 1,6$ (media $\pm \mathrm{DE}$ ) especies $\mathrm{m}^{-2}$. Al comparar el promedio de riqueza de especies por cuadrante se detectaron diferencias significativas entre ambas substratos (Tabla 2).

Los defecaderos presentan índices superiores de diversidad vegetal. La diversidad especifica tuvo un índice promedio de $\mathrm{H}=1,4 \pm 0,39$ (media $\pm \mathrm{DE}$ ) en los defecaderos, con un mínimo de $\mathrm{H}_{\text {mín }}=0,65$ y un máximo de $\mathrm{H}_{\text {máx }}=2,02$. Por su parte, los sitios sin fecas presentaron un índice promedio de $\mathrm{H}=0,48 \pm 0,5$ (media \pm DE), con $\mathrm{H}_{\text {mín }}=0$ y $\mathrm{H}_{\text {máx }}=1,7$. Al compararse los índices de diversidad de ambos tipos de substratos se detectan diferencias estadísticamente significativas (Tabla 2).

La mayoría de las especies presentes en los substratos fueron herbáceas. Solo tres especies fueron leñosas: las arbustivas Gaultheria mucronata y Empetrum rubrum estuvieron presentes solo en los defecaderos, mientras la especie arbórea Nothofagus antarctica se presentó exclusivamente en los sitios sin fecas (Tabla 1). En los sitios abiertos y ausente en los defecade- ros destaca la presencia de Gunnera magellani$c a$, especie fijadora de nitrógeno, con frecuencias promedio de 0,97 presencias $\mathrm{m}^{-2}$ por cuadrante.

Los síndromes de dispersión en los dos tipos de substratos no_presentan un grado de asociación significativo entre sus porcentajes expresados $\left(\mathrm{x}^{2}=14,3 ; \mathrm{P}=0,006\right)$. Por tanto no existe relación entre el tipo de suelo y la frecuencia de los síndromes de dispersión. La dispersión de propágulos por el viento fue el síndrome dominante en la flora total catastrada con un $52 \%$, seguido por la endozoocoria y barocoria ambos con un $19 \%$ (Fig. 2). En los defecaderos las especie de dispersión anemocora y endozoocora no presenta mayor variación representando el 54,6 y $16,7 \%$, respectivamente; al igual que en los suelos sin presencia de fecas en donde los valores fueron similares, 55,6 y 18,2\%.

Los defecaderos presentaron mayores niveles de nutrientes, materia orgánica y agua, que los suelos en sitios desnudos (Tabla 3 ). Todos las variables medidas presentaron diferencias significativas entre ambos substratos, alcanzando valores hasta 10 veces superiores en los defecaderos. El pH en los sitios libres de fecas 
TABLA 2

Valores promedio \pm DE de riqueza de especies e índice de diversidad de Shannon-Wiener para defecaderos y sitios control. Se proporcionan probabilidades de similitud

(prueba U de Mann-Whitney)

Mean \pm SD values of species richness and Shannon-Wiener diversity indices for dung heaps and control sites. Similarity probabilities are provided (Mann-Whitney U-test)

\begin{tabular}{lccc}
\hline Parámetro & Defecaderos $(\mathrm{n}=10)$ & Sitios control $(\mathrm{n}=30)$ & Valor de P \\
\hline Riqueza de especies & $6,8 \pm 2,9$ & $1,70 \pm 1,6$ & $<0,0001$ \\
Índice de diversidad $(\mathrm{H})$ & $1,4 \pm 0,4$ & $0,48 \pm 0,5$ & $<0,0001$ \\
\hline
\end{tabular}

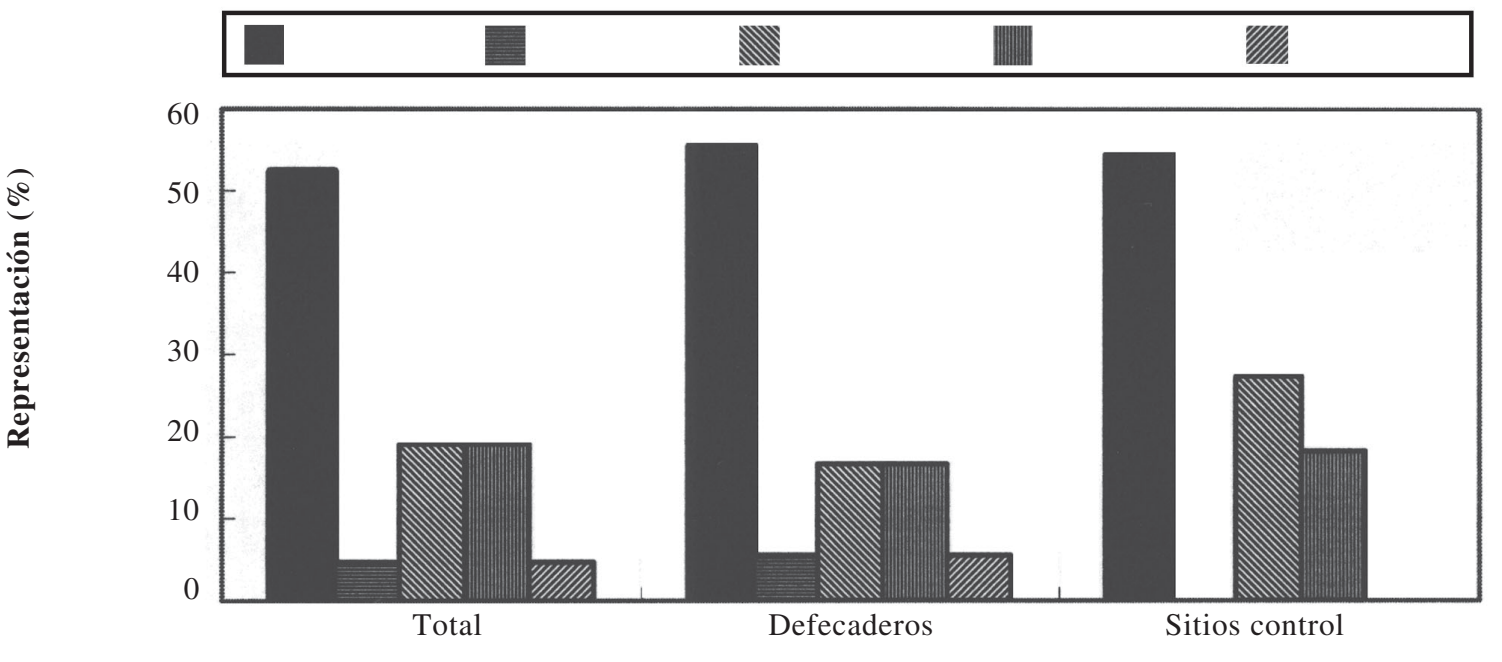

Sitios de estudio

Fig. 2: Representación de los síndromes de dispersión de la flora vascular presente en al área de estudio, defecaderos y sitios control.

Representation of dispersion syndromes in the vascular flora of the study area, comparing camelid dung heaps and control sites.

TABLA 3

Valores promedio \pm DE de las variables de suelo analizadas para cada tipo de substrato.

Se proporcionan probabilidades a partir de la prueba de Wilcoxon $(\mathrm{P})$

Mean values \pm SD) of soil variables for dung heaps and neighbouring substrates.

Wilcoxon test $(\mathrm{P})$ probabilities are provided

\begin{tabular}{|c|c|c|c|}
\hline Parámetros & Defecaderos $(\mathrm{n}=5)$ & Suelos control $(n=5)$ & Valor de P \\
\hline $\mathrm{N}$ disponible $\left(\mathrm{mg} \mathrm{kg}^{-1}\right)$ & $348,30 \pm 31,10$ & $2,53 \pm 1,60$ & 0,0122 \\
\hline P Olsen $\left(\mathrm{mg} \mathrm{kg}^{-1}\right)$ & $205,00 \pm 9,09$ & $2,90 \pm 0,99$ & 0,0122 \\
\hline $\mathrm{K}$ disponible $\left(\mathrm{mg} \mathrm{kg}^{-1}\right)$ & $412,17 \pm 38,18$ & $23,40 \pm 4,98$ & 0,0119 \\
\hline $\mathrm{pH}$ en agua & $7,00 \pm 0,02$ & $6,26 \pm 0,52$ & 0,0111 \\
\hline Materia orgánica (\%) & $81,17 \pm 0,64$ & $0,21 \pm 0,06$ & 0,0119 \\
\hline $\mathrm{K}$ intercambiable $\left(\mathrm{cmol} \mathrm{kg}^{-1}\right)$ & $1,08 \pm 0,09$ & $0,06 \pm 0,01$ & 0,0109 \\
\hline N-Total $(\%)$ & $63,00 \pm 1,41$ & $0,01 \pm 0,07$ & 0,0102 \\
\hline Retención de agua (\%) & $45,08 \pm 2,46$ & $5,46 \pm 1,92$ & 0,0121 \\
\hline
\end{tabular}


presentan una mayor acidez que los defecaderos, a pesar de la orina animal presente en estos últimos.

\section{DISCUSIÓN}

Los trabajos referidos al guanaco en la Patagonia tienen relación con aspectos socio-ecológicos, no analizándose los nexos entre estos animales y la vegetación (Franklin 1982a,b, Fritz 1985, Jurgensen 1985, Sarno et al. 1999). En este contexto, los defecaderos solo han sido analizados en función de estudios poblacionales (comportamiento, territorialidad). Efectos de los defecaderos sobre la abundancia de las especies vegetales han sido observados pero no cuantificados en pastizales de Tierra del Fuego (Franklin 1982a,b) y del Parque Torres del Paine (M.Ortega comunicación personal). Los resultados aquí expuestos muestran que la presencia de defecaderos de guanacos ocasionan un efecto positivo sobre la riqueza y diversidad de las plantas vasculares presentes en una intermorrena lateral sucesionalmente temprana.

Parte del efecto positivo de los defecaderos sobre las plantas vasculares podría deberse al rol dispersivo de los guanacos. Se ha observado la presencia de semillas en las fecas de guanacos (J.M. Henríquez observaciones personales) pero no ha sido documentada ni analizada su composición ni viabilidad. Sin embargo, el análisis de los síndromes de dispersión muestra que la endozoocoria presenta bajos porcentajes de representación en la flora total siendo poco probable explicar la riqueza de especies y el proceso de colonización a partir de este medio. Considerando la mayor riqueza de especies presentes en los defecaderos con relación a los sitios control, es posible considerar que sea mayor el número de especies palatables no clasificadas como endozoocoras cuyas semillas podrían sobrevivir a la ingestión de los herbívoros. Al respecto, estudios recientes concluyen que probablemente la endozoocoria, en general, juegue un rol más importante en la dispersión de semillas que la documentada a la fecha (Gardener et al. 1993a, 1993b, Welch 1995, Gill \& Beardall 2001, Pakeman et al. 2002).

El efecto positivo de los defecaderos podría deberse a la oferta de condiciones más favorables para el establecimiento de plántulas. Los análisis de suelos y fecas (Tabla 3) indican que los defecaderos proporcionan cantidades mayores de nutrientes y materia orgánica disponible para el desarrollo de la vegetación. Además, el mayor porcentaje de las especies presentes en el área de estudio son anemocoras (52 \%), resultado coincidente con lo indicado por Dollenz (1991) para el Glaciar Dickson, en el Parque Torres del Paine (Chile).

Los sitios sucesionalmente tempranos, como la geoforma estudiada, se caracterizan por disponibilidades muy bajas de nutrientes esenciales, sobre todo el nitrógeno (Matthews 1992, Chapin et al 1994) y suelos descubiertos con una fuerte exposición a la radiación solar, lo cual provoca grandes fluctuaciones en la temperatura del suelo y una rápida desecación de la superficie (Chapin 1993). Diversos estudios han señalado que las especies que crecen sobre fecas se ven favorecidas por la riqueza de nutrientes y materia orgánica (Tilman 1988, Matthews 1992, Chapin et al 1994, Rousset \& Lepart 1999), y las plántulas son favorecidas por la presencia de nutrientes y la protección a la desecación (Malo \& Suárez 1998).

Las observaciones en terreno, referidas al comportamiento del guanaco, sugieren que las entradas de propágulos y nutrientes al sistema son escasas producto del número limitado de ejemplares (población residente no supera los tres individuos) y la baja densidad de defecaderos $\left(0,375\right.$ ha $^{-1}$ inferior a los 0,5 ha $^{-1}$ dada para Tierra del Fuego (Franklin 1982b)). La baja densidad poblacional observada probablemente minimiza los efectos tóxicos de las fecas y orina en los defecaderos favoreciendo la expresión de la vegetación sobre ellos. En contraste, en altas densidades poblacionales los efectos tóxicos de la feca fresca limitan el establecimiento y desarrollo de la vegetación a los bordes de los defecaderos (Raedeke 1979, Franklin 1982a).

Por otro lado, la baja densidad de defecaderos es explicable al considerar lo dinámica del terreno, expuesto a deshielos, derrumbes y socavamientos. La acción de marcar su territorio lleva a los guanacos a crear nuevos defecaderos solo cuando nuevos sitios son denudados, pero manteniendo actividad en los antiguos. Sin embargo, a pesar de que los defecaderos son visitados anualmente, nuevos y antiguos, producto del limitado número de individuos, el aporte de fecas es escaso en volumen y su expansión en superficie es mínima.

Jumpponen et al. (1999), en terrenos recientemente expuestos, encontraron que el establecimiento y sobrevivencia de plantas individuales no es aleatoria y ocurre solo en "sitios seguros" (Harper et al. 1961), los cuales pueden ser caracterizados simplemente por sus atributos físicos, o por las disponibilidades de recursos. Aunque la entrada de propágulos y aporte de nutrientes por los guanacos sea muy localizada, con un im- 
pacto menor sobre el sistema total, los defecaderos ejercerían una influencia positiva en la dinámica sucesional de los terrenos recientemente desglaciados del valle estudiado, principalmente por ser una fuente cercana y constante de semillas. Los resultados permiten concluir que los defecaderos de guanacos actúan como micrositios o sitios seguros (Harper et al. 1961) con condiciones favorables para la germinación y establecimiento vegetal de las semillas presenten en las fecas o de las que arriban, produciendo el aumento de la abundancia y diversidad de las plantas vasculares presentes en terrenos recientemente derretidos.

\section{AGRADECIMIENTOS}

El autor agradece el financiamiento de FONDECYT al proyecto 2000134. De manera particular a la III Zona Naval de la Armada de Chile y Gobernación Marítima de Magallanes, por el transporte marítimo facilitado, sin lo cual hubiese sido imposible acceder al área de estudio. A Ernesto Teneb, Cristina Furrianca y Claudia Mansilla por la valiosa ayuda prestada en la toma de datos. Al Dr. Chris Lusk y a dos revisores anónimos por la revisión crítica del manuscrito.

\section{LITERATURA CITADA}

CHAPIN FS III (1993) Physiological controls over plant establishment in primary succession. En: Miles J \& DWH Walton. Primary succession on land: 161.178. Blackwell Scientific Publications, Oxford, United Kingdom.

CHAPIN FS III, LR WALKER, CL FASTIE \& LC SHARMAN (1994) Mechanisms of primary succession following deglaciation at Glacier Bay, Alaska. Ecological Monographs 64: 149-175.

CROCKER RL \& J MAJOR (1955) Soil development in relation to vegetation and surface age at Glacier Bay, Alaska. Journal of Ecology 43: 427-448.

DOLLENZ O (1991) Sucesión vegetal en el sistema morrénico del Glaciar Dickson, Magallanes, Chile. Anales del Instituto de la Patagonia. Serie Ciencias Naturales (Chile) 20: 49-60.

EDWARDS PJ \& MP GILLMAN (1987) Herbivores and plant succession. En: Gray AJ, MJ Crawley \& PJ Edwards (eds) Colonization, succession and stability: 295-314. Blackwell Scientific Publications, Oxford, United Kingdom

FRANKLIN WL (1982a) Lama language: modes of communication in the South American camelids. Lama World 1: 6-11.

FRANKLIN WL (1982b) Constrasting socioecologies of South America's wild camelids: the vicuña and guanaco. En: Eisenberg JF \& D Kleiman (eds) Advances in the study of mammalian behavior: 573-629. American Society of Mammalogists, Special Publications 7: $1-753$.
FRITZ MA (1985) Population dynamics and preliminary estimates of the harvestibiliy of the Patagonian guanaco. MS Thesis, Iowa State University, Ames, Iowa, USA. $61 \mathrm{pp}$.

GARDENER CJ, JG McIVOR \& A JANSEN (1993a) Passage of legume and grass seeds through the digestive tract of cattle and their survival in faeces. Journal of Applied Ecology 30: 63-74.

GARDENER CJ, JG MCIVOR \& A JANSEN (1993b) Survival of seeds of tropical grassland species subjected to bovine digestion. Journal of Applied Ecology 30: $75-85$.

GILL RMA \& V BEARDALL (2001) The impact of deer on woodlands: the effects of browsing and seed dispersal on vegetation structure and composition. Forestry 74: 209-218.

HARPER JL, JN CLATWORTHY, IH MCNAUHTON \& GS SAGAR (1961) The evolution of closely related species living in the same area. Evolution 15: 209-227.

HENRÍQUEZ JM, E PISANO \& C MARTICORENA (1995) Catálogo de la flora vascular de Magallanes (XII Región), Chile. Anales del Instituto de la Patagonia. Serie Ciencias Naturales (Chile) 23: 5-30.

HOWE H \& L WESTLEY (1986) Ecology of pollination and seed dispersal. En: Crawley M (ed) Plant ecology: 185-216. Blackwell, Oxford, United Kingdom.

INTERNATIONAL ORGANIZATION FOR STANDARDIZATION (ISO) (1993) Soil quality: determination of dry matter and water content on a mass basis - Gravimetric method. ISO 11465, Geneva, Switzerland. 3 pp.

INTERNATIONAL ORGANIZATION FOR STANDARDIZATION (ISO) (1994) Soil quality: determination of pH. ISO 10390, Geneva, Switzerland. 5 pp.

JUMPPONEN A, H VARE, K MATTSON, R OHTONEN \& J TRAPPE (1999) Characterization of "safe sites" for pioneers in primary succesion on recently deglaciated terrain. Journal of Ecology 87: 98-105.

JURGENSEN TE (1985) Seasonal territoriality in a migratory guanaco population. MS Thesis, Iowa State University, Ames, Iowa, USA. 54 pp.

LONGERI L, J ETCHEVERS \& J VENEGAS (1979) metodología de perfusión para estudios de nitrificación en suelos. Ciencia e Investigación Agraria (Chile) 6:295-299.

MALO JE \& E SUAREZ (1995) Establishment of pasture species on cattle dung: the role of endozoochorous seeds. Journal Vegetation Science 6: 169-174.

MALO JE \& E SUAREZ (1998) The dispersal of a dryfruited shrub by red deer in a Mediterranean ecosystem. Ecography 21: 204-211.

MATTHEWS JA (1992) The ecology of recently-deglaciated terrain: A geoecological approach to glacier forest lands and primary succession. Cambridge University Press, Cambridge, United Kingdom. 336 pp.

MEADOWS PS \& A MEADOWS (eds) (1991) The environmental impact of burrowing animals and animal burrows. Clarendon Press, Oxford, United Kingdom. 267 pp.

NORMA CHILENA (1999) Suelos - Determinación del fósforo extraíble con solución de bicarbonato de sodio. Sociedad Chilena de la ciencia del suelo. NCh 2058, Santiago, Chile. 2 pp.

PAKEMAN RJ, G DIGNEFFE \& JL SMALL (2002) Ecologycal correlates of endozoochory by herbivores. Funtional Ecology 16: 296-304.

PICKETT STA \& PS WHITE (1985) The ecology of natural disturbance and patch dynamics. Academic Press, Orlando, Florida, USA. 472 pp.

PISANO E (1971) Comunidades vegetales del área del fiordo Parry. Anales Instituto de la Patagonia 2: 76-92. 
RAEDEKE K (1978) El guanaco de Magallanes, Chile. Su distribución y biología. Ministerio de Agricultura de Chile, Corporación Nacional Forestal (CONAF), Chile, Publicación Técnica 4: 1-182.

RAEDEKE K (1979) Population dynamics and socioecology of the guanaco (Lama guanicoe) of Magallanes, Chile. Ph.D. Dissertation, University. Washington, Seattle, USA. 409 pp.

ROBARGE WP, A EDWARDS \& B JAHNSON (1983) Water and waste water analysis for nitrate via nitration of salicylic acid. Communication Soil Science and Plant Analysis 14: 1207-1215.

ROUSSET O \& J LAPART (1999) Shrub facilitation of Quercus humilis regeneration in succession on calcareous grasslands. Journal Vegetation Science 10: 493-502.

SARNO RJ, WR CLARK, MS BANK, WF PREXL, MJ BEHL, WE JOHNSON \& WL FRANKLIN (1999) Juvenile guanaco survival: management and conservation implications. Journal of Applied Ecology 36: 937-945.

SCHULTE EE (1995) Recommended soil organic matter tests. In: Horton ML (ed) Ecommended soil testing procedures for the northeastern United States. Se- cond edition. Northeastern Regional Publication $\mathrm{N}^{\mathrm{o}}$ 493, Chapter 8: 52-61. Disponible en: http:// bluehen.ags.udel.edu/deces/prod_agric/chap495.htm.

TILMAN GD (1988) Plant strategies and the dynamics and structure of plant communities. Princeton Monographs. Princeton, New Jersey, USA. 360 pp.

UNITED STATES DEPARTMENT OF AGRICULTURE (1996) Soil survey laboratory methods manual. Soil Survey Investigations Report $\mathrm{N}^{\circ}$ 42. Version 3.0. Washington, District of Columbia, USA. 693p.

VILLAGRÁN C, J ARMESTO \& P LEIVA (1986) Recolonización postglacial de Chiloé insular: evidencias basadas en la distribución geográfica y los modos de dispersión de la flora. Revista Chilena de Historia Natural 59: 19-39.

WALKER LR \& FS III CHAPIN (1987) Interactions among processes controlling succesional change. Oikos 50: 131-135.

WELCH D (1985) Studies in the grazing of heather moorland in north-east Scotland. IV. Seed dispersal and plant establishment in dung. Journal of Applied Ecology 22: 461-472. 